\title{
The effect of music therapy on pain, anxiety and depression in patients after coronary artery bypass grafting
}

\author{
Wang-Sheng Dai ${ }^{1 \dagger}$, Shu-Ting Huang ${ }^{1,2 \dagger}$, Ning $\mathrm{Xu}^{1,2}$, Qiang Chen ${ }^{1,2^{*}}$ (D) and Hua Cao ${ }^{1 *}$
}

\begin{abstract}
Objective: The purpose of this study was to explore the effects of music therapy on pain, anxiety and depression in patients after coronary artery bypass grafting.

Methods: A retrospective study of 99 patients after coronary artery bypass from January 2017 to January 2019 was conducted in a cardiac center in China. According to the different interventions, all the participants were divided into 3 groups: group A: music therapy; group B: rest without music therapy; and group C: conventional treatment. The Numerical Rating Scale (NRS), Self-Rating Anxiety Scale (SAS) and Self-Rating Depression Scale (SDS) were used to measure the patient's pain, anxiety and depression before and after $30 \mathrm{~min}$ of the intervention.

Results: There were no significant differences in the NRS, SDS and SAS scores between the three groups of patients before the intervention. After 30 min of music therapy, the NRS, SDS and SAS scores of patients in group A were significantly lower than those before music therapy, and the differences were statistically significant. However, before and after the intervention in groups B and C, the NRS, SDS and SAS scores were not statistically significant. By comparison among the three groups after 30 min of intervention, the NRS, SDS and SAS scores in patients in group A were significantly lower than those in groups B and C, and the differences were statistically significant. The scores were not significantly different between groups $B$ and $C$.
\end{abstract}

Conclusion: Music therapy can effectively alleviate the pain, anxiety and depression of patients after coronary artery bypass grafting.

Keywords: Music therapy, CABG, Pain, Anxiety, Depression

\section{Introduction}

Coronary atherosclerotic heart disease is one of the most common heart diseases in elderly individuals. With the development of minimally invasive technology and equipment, many coronary heart diseases can be treated with minimally invasive treatment, including percutaneous coronary intervention, coronary artery bypass

\footnotetext{
*Correspondence: chenqiang2228@163.com; caohua0791@163.com

tWang-Sheng Dai and Shu-Ting Huang contributed equally to this work. 'Department of Cardiac Surgery, Fujian Maternity and Child Health Hospital, Affiliated Hospital of Fujian Medical University, Fuzhou, China

Full list of author information is available at the end of the article
}

grafting with a small left anterior lateral thoracotomy, robotic off-pump coronary artery bypass grafting, etc. However, coronary artery bypass grafting with median thoracotomy is still the main surgical method for the treatment of severe and multiple coronary artery disease [1]. Surgery requires midline splitting of the sternum, a large incision and an indwelling drainage tube; thus, patients usually suffer from obvious postoperative pain [2]. Studies have shown that more than $80 \%$ of patients suffer from moderate to severe postoperative pain [3]. Postoperative patients need to be placed in an unfamiliar environment with $24 \mathrm{~h}$ of artificial lighting with possible 
accompanying medical instrument monitoring, no family support, and worries about the success of the operation, all of which can easily make the patients feel anxious and depressed. Although the problems of postoperative pain, anxiety and depression have been taken seriously, the management and treatment are still incomplete $[4$, 5]. This study summarized our experience in implementing music therapy for patients after coronary artery bypass grafting and evaluated its effects on improving patients' postoperative pain, anxiety, and depression.

\section{Information and methods}

\section{Normal information}

According to the pain of the three groups of patients in the pilot test, assuming a difference of $10 \%$ between the three independent populations, $\alpha=0.05, \beta=0.2$, it was calculated that 30 participants were required for each group. Assuming a $10 \%$ dropout rate, the total sample size required was 99 cases (33 cases per group), which were some of the cases at the hospital at this period. The study was approved by the ethics committee of our university, and written informed consent was obtained from each participant.

This study was a retrospective study conducted by a cardiac hospital. The clinical data of 99 patients who underwent coronary artery bypass graft from January 2017 to January 2019 were collected and divided into 3 groups according to the selected different types of intervention: group A: music therapy group; group B: rest without music therapy group; and group C: conventional treatment group. All of the patients underwent routine surgery with or without cardiopulmonary bypass. The following inclusion criteria were used: tracheal intubation was removed on same the day as the surgery or the next day, hemodynamics were stable, the patient was transferred to the general ward 1 to 2 days after surgery, the analgesia pump was stopped, the patient suffered no hearing impairment, and the patient was able to cooperate with the treatment and this research. The exclusion criteria were as follows: 1 , the patient had a history of chronic pain, mental disorders, or hearing impairment, 2 , the patient's condition was combined with other important organ dysfunction, 3 , the patient experienced severe complications during the perioperative period, 4 , the patient was unable to cooperate with this research or was unwilling to sign the informed consent, and 5, the patient used any painkillers or other methods to reduce pain before our intervention.

\section{Methods}

The patients were lying on their bed in the general ward at 20:00. The surrounding environment was quiet, comfortable, safe, and the light was soft. The bed curtains were pulled closed, and the patients had no interference from the medical staff or researchers. All patients wore binaural headphones. Music was chosen and played in group A for $30 \mathrm{~min}$. Patients could choose their favorite music with the volume based on the their comfort. There was no restriction on the type of music, but mostly light music and relaxing music. The patients had just a quiet rest for $30 \mathrm{~min}$ without playing music in group $\mathrm{B}$. The patients in group $\mathrm{C}$ were given routine treatment with wearing headphones for $30 \mathrm{~min}$, and normal activities were performed.

\section{Research tool}

Numerical Rating Scale (NRS): This scale has been widely used in pain-related studies with good reliability and validity [6]. The scale uses numbers instead of text to indicate the degree of pain, segmented on a straight line, with the degree of pain expressed with 11 numbers from 0 to 10.0 means no pain, $1-3$ points means mild pain ( 1 point: no pain in a quiet supine position, pain when turning over and coughing; 2 points: pain when coughing, no pain in deep breathing; 3 points: no pain in a quiet supine, pain with deep breathing and coughing), 4-6 points means moderate pain (4 points: gap pain when in a quiet supine position; 5 points: persistent pain when in a quiet supine position; 6 points: severe pain when in a quiet supine position), 7-10 points means significant pain ( 7 points: heavier pain, upset, unable to fall asleep; 8 points: persistent, intolerable pain, sweating throughout the body; 9 points: severe, intolerable pain; 10 points: the most painful, life was worse than death).

Self-Rating Depression Scale (SDS): The Zung SelfRating Depression Scale was used. The SDS has been widely used in clinical practice and has high reliability and validity [7]. The scale consists of 20 items with 4 scoring grades and includes 10 negative symptoms and 10 positive symptoms. Each question represents the characteristics of depression. All of the items reflect mood, physical discomfort, mental activity, behavior and psychological symptoms. According to the frequency of positive symptoms, numbers from 1 to 4 are used for scoring. Based on the frequency of negative symptoms, a rough score is obtained using the reverse score method (4 to 1 ). The standard score is the score multiplied by 1.25 and is rounded off. The upper limit score is 41 , and the standard score is 53 . The higher the score, the more pronounced the depression tendency.

Self-Rating Anxiety Scale (SAS): The Zung Self-Rating Anxiety Scale was used. The SAS has been widely used in clinical practice and has high reliability and validity [8]. Fifteen items are expressed as negative words, and scores are based on the frequency of symptoms (1 to 4 ). Affirmative terms are used to indicate 5 items, and according to the frequency of symptoms, the reverse scoring method (4 to 1 ) is used for scoring. The scores of all 
items are summed to obtain the total score. The standard score is multiplied by 1.25 and rounded off. The standard score average is 50. Score description: $<50$ means normal, 50 59 means mild anxiety, $60 \sim 69$ means moderate anxiety, and $\geq 70$ means severe anxiety.

\section{Data collection}

The SAS, SDS and NRS scores were recorded separately before and after $30 \mathrm{~min}$ of the intervention to measure the patient's pain, anxiety and depression. Participants completed the questionnaire independently; the researchers were not allowed to interfere with the participants' decisions but were able to explain the questions to the participants or provided language translation. After all questionnaires were completed, the data were statistically analyzed by specialized independent researchers.

\section{Statistical analysis}

Quantitative data are expressed as the means \pm standard deviations, and continuous data were compared between groups by analysis of variance; qualitative data were compared between groups by chi-square test; $P<0.05$ was considered statistically significant.

\section{Results}

The general information of all patients is shown in Table 1. There were no significant differences among the three groups of patients. The NRS, SAS and SDS scores before the intervention were also not statistically significant. These results indicated that the three groups of patients were homogeneous; thus, the data were comparable between groups. After $30 \mathrm{~min}$ of music therapy, the NRS, SDS and SAS scores of patients in group A were significantly lower than those before music therapy, and the differences were statistically significant $(P<$ 0.05). However, before and after intervention in groups $B$ and $C$, the NRS, SDS and SAS scores were not statistically significant. After 30 min of intervention, the NRS, SDS, and SAS scores in patients in group A were significantly lower than those in groups $\mathrm{B}$ and $\mathrm{C}$, and the differences were statistically significant $(P<0.05)$. The scores were not significantly different between groups $B$ and $C$. These results indicated that music therapy could significantly reduce the NRS, SDS and SAS scores of patients after coronary artery bypass grafting (Table 2).

\section{Discussion}

Pain is an unpleasant feeling and emotional experience related to tissue damage or potential tissue damage. It is one of the most common symptoms of surgical patients

Table 1 Comparison of general information of the three groups of patients

\begin{tabular}{|c|c|c|c|c|}
\hline Variable & Group A & Group B & Group C & $P$ value \\
\hline Age & $53.4 \pm 12.6$ & $55.8 \pm 10.9$ & $53.1 \pm 14.8$ & 0.547 \\
\hline Male/female & $18 / 15$ & $17 / 16$ & $19 / 14$ & 0.885 \\
\hline \multicolumn{5}{|l|}{ Number of bridging vessels } \\
\hline one & 5 & 4 & 3 & \multirow[t]{3}{*}{0.932} \\
\hline two & 8 & 7 & 9 & \\
\hline three or more & 20 & 22 & 21 & \\
\hline \multicolumn{5}{|l|}{ NYHA } \\
\hline I & 0 & 0 & 0 & \multirow[t]{4}{*}{0.563} \\
\hline$\|$ & 23 & 25 & 21 & \\
\hline III & 10 & 8 & 12 & \\
\hline IV & 0 & 0 & 0 & \\
\hline LVEF & $56.9 \pm 11.5$ & $58.2 \pm 9.3$ & $55.8 \pm 12.7$ & 0.612 \\
\hline Incision length & $13.1 \pm 2.8$ & $14.8 \pm 2.1$ & $14.5 \pm 2.8$ & 0.886 \\
\hline \multicolumn{5}{|l|}{ Marriage } \\
\hline married & 30 & 31 & 30 & \multirow[t]{3}{*}{0.906} \\
\hline spinsterhood & 1 & 1 & 2 & \\
\hline widowed & 2 & 1 & 1 & \\
\hline \multicolumn{5}{|l|}{ Education } \\
\hline Middle school and below & 16 & 18 & 15 & \multirow[t]{3}{*}{0.719} \\
\hline High school and technical secondary school & 14 & 10 & 15 & \\
\hline College and above & 3 & 5 & 3 & \\
\hline
\end{tabular}


Table 2 Comparison of NRS, SDS, and SAS scores between the three groups of patients before and after intervention

\begin{tabular}{lllll}
\hline Scores & Group A & Group B & Group C & P value \\
\hline NRS & & & & \\
pretest & $7.3 \pm 1.9$ & $7.1 \pm 1.6$ & $7.2 \pm 2.0$ & 0.832 \\
posttest & $4.2 \pm 2.1^{*}$ & $7.2 \pm 1.8^{\#}$ & $7.4 \pm 1.9^{\#}$ & 0.013 \\
SDS & & & & \\
pretest & $58.6 \pm 10.4$ & $60.8 \pm 12.8$ & $62.2 \pm 13.6$ & 0.761 \\
posttest & $46.3 \pm 9.3^{*}$ & $59.3 \pm 14.1^{\#}$ & $62.9 \pm 11.8^{\#}$ & 0.022 \\
SAS & & & & \\
pretest & $64.6 \pm 13.8$ & $63.2 \pm 14.9$ & $62.1 \pm 12.7$ & 0.846 \\
posttest & $54.3 \pm 11.6^{*}$ & $60.5 \pm 15.5^{\#}$ & $62.6 \pm 13.9^{\#}$ & 0.034 \\
\hline
\end{tabular}

\# means $P<0.05$ compared with group $A$

* means $P<0.05$ compared with before music therapy

and affects the recovery of local or overall functions, which can even threaten the patient's life $[9,10]$. Especially for patients with severe coronary heart disease, coronary artery bypass grafting must be performed with a median sternal incision. As the chest cavity moves with breathing and coughing, the cut edges of the sternum will rub against each other, causing severe pain. If the pain cannot be effectively controlled, the patient's concentrations of catecholamines will increase, which will lead to increased blood pressure, a faster heartbeat, increased peripheral vascular resistance, and increased incidence of postoperative bleeding and will affect the patient's breathing, cough, mood, and appetite. The corresponding results can delay the patient's time to get out of bed, affect the patient's postoperative recovery, and even increase the incidence of complications. Prolonged pain can also easily cause patients to have negative emotions, such as anxiety, anxiety, nervousness and even depression $[11,12]$. These effects are more pronounced in older people with weak constitutions who undergo coronary artery bypass grafting. In recent years, increasing amounts of attention have been paid to pain management in these types of older patients. Thus, how to effectively reduce postoperative pain and improve the psychological state of postoperative patients has become a research hotspot.

Although the current treatment of postoperative pain is mainly focused on drug treatment, increasing numbers of nondrug treatment methods have also begun to be widely used [13, 14]. Music therapy is an innovative nondrug treatment that can stimulate the cerebral cortex to reduce stress-related hormones and can have a positive effect on improving emotions through neuroendocrine pathways [15]. It can also have a direct impact on the brain edge and the brainstem reticular structure of the nervous system, reducing sympathetic nervous system activity and increasing parasympathetic neural activity, reducing the body's physiological response to stress and lowering the cortisol level in the patient's body, thereby stabilizing the patient's blood pressure and heart rate under stress [16]. Music therapy relieves pain by distraction and improves the emotional dimension of pain by affecting emotions and feelings. Many domestic and overseas research reports have confirmed that music intervention can have significant effects on pain relief and emotional stability and can relieve stress and anxiety. A clinical randomized controlled trial conducted by Ko SY and his colleagues showed that relaxed music could improve the pain and anxiety of patients undergoing colonoscopy and improve treatment satisfaction [17]. A study by Yaman Aktaş et al. showed that music therapy could be used as a nondrug therapy to reduce pain in patients with mechanical ventilation [18]. Ernsten and his teams found that perioperative music therapy could effectively relieve acute pain associated with surgery [19]. Rohilla et al. showed that music therapy could help reduce anxiety, pain and the use of opioids during surgical dressing changes in burn patients [20]. Music therapy can relieve the degree of postoperative pain in surgical patients and can be beneficial in stabilizing the patient's heart rhythm and maintaining the hemodynamic balance, as shown in a study by Chen and her colleagues [21]. Music can adjust and balance people's emotions. It can slow down the heart rate and dilate the blood vessels, thereby improving the blood circulation, reducing the heart load, removing the metabolic products produced by myocardial hypoxia, and consequently alleviating angina pectoris [22]. In this study, the pain score of patients in the music therapy group was significantly lower than that of patients who did not receive music therapy and that of the preintervention status; this study also confirmed that music therapy could relieve pain in patients after coronary artery bypass grafting.

In addition, music also had a good sedative effect [23, 24]. Music stimulated the right hemisphere limbic system, which manages emotions, feelings and sensory centers to bring about a sedation effect and improve the patient's negative emotions. When patients listen to comfortable and sweet-sounding music, they produce pleasant associations, which can create a comfortable and satisfied mood to relieve anxiety. This study also showed that the SAS and SDS scores of the music therapy group after music therapy were significantly lower than those before the music therapy, and the SAS and SDS scores of the music therapy group were significantly lower than those of the non-music therapy group. Such results indicated that listening to comfortable and pleasant music could significantly relieve the patients' anxiety and depression.

The study still had some shortcomings. First, this was a single-center retrospective study, not a randomized controlled study; thus, there was a certain deviation in 
the selection of cases, but the findings still had certain clinical significance. In addition, this was a single-center study with a relatively small sample size. A multicenter and large-sample study will be performed in the future. Third, this study was only limited to specific patients undergoing coronary artery bypass surgery. Whether music therapy had the same effect in other patients undergoing other types of surgery will require further study.

\section{Conclusion}

Music therapy as a nondrug treatment method is very safe, simple and economical to implement and can effectively alleviate the pain, anxiety and depression of patients undergoing coronary artery bypass grafting. Thus, it is worthy of clinical promotion and application.

\section{Abbreviations}

NRS: Numerical Rating Scale; SAS: Self-Rating Anxiety Scale; SDS: Self-Rating Depression Scale

\section{Acknowledgements}

We highly acknowledge the contribution by the participating doctors: DaoZhong Chen, Liang-Wan Chen, Feng Lin, Qi-Min Wang, Han-Fan Qiu, XueShan Huang, Dong-Shan Liao, Xiao-Fu Dai, Zeng-Chun Wang.

\section{Authors' contributions}

Qiang Chen and Hua Cao designed the study and submitted the manuscript. Wang-Sheng Dai, Shu-Ting Huang and Ning Xu collected and analyzed data together. Wang-Sheng Dai drafted the article. All authors read the final version of this article and approved for publication.

\section{Funding}

No.

\section{Availability of data and materials}

Data sharing not applicable to this article as no data sets were generated or analyzed during the current study.

\section{Ethics approval and consent to participate}

The present study was approved by the ethics committee of Fujian Medical University, China and adhered to the tenets of the Declaration of Helsinki.

\section{Consent for publication}

Not applicable.

\section{Competing interests}

All authors declare that they have no competing interests.

\section{Author details}

${ }^{1}$ Department of Cardiac Surgery, Fujian Maternity and Child Health Hospital, Affiliated Hospital of Fujian Medical University, Fuzhou, China. ${ }^{2}$ Department of Cardiovascular Surgery, Union Hospital, Fujian Medical University, Fuzhou, China.

Received: 24 January 2020 Accepted: 4 May 2020

Published online: 11 May 2020

\section{References}

1. Head SJ, Milojevic M, Daemen J, Ahn JM, Boersma E, Christiansen EH, Domanski MJ, Farkouh ME, Flather M, Fuster V, Hlatky MA, Holm NR, Hueb WA, Kamalesh M, Kim YH, Mäkikallio T, Mohr FW, Papageorgiou G, Park SJ, Rodriguez AE, Sabik JF 3rd, Stables RH, Stone GW, Serruys PW, Kappetein AP. Mortality after coronary artery bypass grafting versus percutaneous coronary intervention with stenting for coronary artery disease: a pooled analysis of individual patient data. Lancet. 2018;391(10124):939-48.
2. Goodwin MJ, Bissett L, Mason P, Kates R, Weber J. Early extubation and early activity after open heart surgery. Crit Care Nurse. 1999 Oct;19(5):18-26.

3. Allred KD, Byers JF, Sole ML. The effect of music on postoperative pain and anxiety. Pain Manag Nurs. 2010;11(1):15-25.

4. Sendelbach SE, Halm MA, Doran KA, Miller EH, Gaillard P. Effects of music therapy on physiological and psychological outcomes for patients undergoing cardiac surgery. J Cardiovasc Nurs. 2006;21(3):194-200.

5. Mueller XM, Tinguely F, Tevaearai HT, Revelly JP, Chioléro R, von Segesser LK. Pain location, distribution, and intensity after cardiac surgery. Chest. 2000;118(2):391-6.

6. Gagliese L, Weizblit N, Ellis W, Chan WW. The measurement of postoperative pain: a comparison of intensity scales in younger and older surgical patients. Pain. 2005;117(3):412-20.

7. Zung WW. A self-rating depression scale. Arch Gen Psychiatry. 1965;12:6370.

8. Zung WW. A rating instrument for anxiety disorders. Psychosomatics. 1971; 12(6):371-9.

9. Apfelbaum JL, Chen C, Mehta SS, Gan TJ. Postoperative pain experience: results form a national survey suggest postoperative pain continues to be undermanaged. Anesth Analg. 2003;97(2):534-40.

10. Tse MMY, Chan MF, Benzie IF. The effect of music therapy on postoperative pain, heart rate, systolic blood pressures and analgesic use following nasal surgery. J Pain Palliat Care Pharmacother. 2005;19(3):21-9.

11. Evangelism JA, Parsons M, Renneburg AK. Chest pain in children: diagnosis through history and physical examination. J Pediatr Health Care. 2000;14(1): $3-8$

12. Kehletc $H$. The stress response to surgery: release mechanisms and the modifying effect of pain relief. Acta Chir Scand Suppl. 1989;550:22-8.

13. Zimmerman L, Nieveen J, Barnason S, Schmaderer M. The effects of music interventions on postoperative pain and sleep in coronary artery bypass graft (CABG) patients. Sch Inq Nurs Pract. 1996;10(2):153-70 discussion 1714.

14. Nilsson U, Rawal N, Enqvist B, Unosson M. Analgesia following music and therapeutic suggestions in the PACU in ambulatory surgery; a randomized controlled trial. Acta Anaesthesiol Scand. 2003;47(3):278-83.

15. Stegemann T, Geretsegger M, Quoc EP, Riedl H, Smetana M. Music therapy and other music-based interventions in pediatric health care: an overview. Medicines (Basel). 2019;6(1):25.

16. Twiss E, Seaver J, McCaffrey R. The effect of music listening on older adults undergoing cardiovascular surgery. Nurs Crit Care. 2006;11(5):224-31.

17. Ko SY, Leung DY, Wong EM. Effcets of easy listening music intervention on satisfaction, anxiety, and pain in patients undergoing colonoscopy: a pilot randomized controlled trial. Clin Interv Aging. 2019;14:977-86.

18. Yaman Aktas $Y$, Karabulut N. Relief of procedural pain in critically III patients by music therapy: a randomized controlled trial. Complement Med Res. 2019:26(3):156-65.

19. Ernsten L, Hepp P, Fehm T, Schaal NK. Perioperative music-induced analgesia: comparison of the effect of music on pain between preoperative, intraoperative and postoperative application. Schmerz. 2019;33(2):100-5.

20. Rohilla L, Agnihotri M, Trehan SK, Sharma RK, Ghai S. Effect of music therapy on pain perception, anxiety, and opioid use during dressing change among patients with burns in India: a quasi-experimental, cross-over pilot study. Ostomy Wound Manage. 2018;64(10):40-6.

21. Chan MF, Wong OC, Chan HL, Fong MC, Lai SY, Lo CW, Ho SM, Ng SY, Leung SK. Effects of music on patients undergoing a C-clamp procedure after percutaneous coronary interventions. J Adv Nurs. 2006;53(6):669-79.

22. McCaffrey $\mathrm{R}$, Taylor $\mathrm{N}$. Effective anxiety treatment prior to diagnostic cardiac catheterization. Holist Nurs Pract. 2005;19(2):70-3 Review.

23. Voss JA, Good M, Yates B, Baun MM, Thompson A, Hertzog M. Sedative music reduces anxiety and pain during chair rest after open-heart surgery. Pain. 2004;112(1-2):197-203.

24. Ortega A, Gauna F, Munoz D, Oberreuter G, Breinbauer HA, Carrasco L. Music therapy for pain and anxiety Management in Nasal Bone Fracture Reduction: randomized controlled clinical trial. Otolaryngol Head Neck Surg. 2019;161(4):613-9.

\section{Publisher's Note}

Springer Nature remains neutral with regard to jurisdictional claims in published maps and institutional affiliations. 